\title{
Cryoballoon pulmonary vein isolation as a standard approach for interventional treatment of atrial fibrillation. A review and a practical guide to an effective and safe procedure
}

\author{
Grzegorz Kiełbasa, Marek Jastrzębski \\ First Department of Cardiology, Interventional Electrocardiology and Hypertension, Jagiellonian University Medical College, Krakow, Poland
}

Adv Interv Cardiol 2020; 16, 4 (62): 359-375

DOI: https://doi.org/10.5114/aic.2020.101760

\begin{abstract}
A bstract
Since the cryoballoon was introduced into clinical practice, approximately half a million patients have undergone a pulmonary vein isolation (PVI) using this tool throughout the world. This single-shot technique makes the pulmonary vein isolation procedure easier and has the potential to expand access to the interventional treatment of atrial fibrillation (AF), eventually leading to a reduction of the AF-related disease burden. Several studies and metanalyses have assessed the acute and long-term efficacy of cryoballoon-based PVI. The reported success rate of PV isolation during the procedure is about $98 \%$. Despite this, the long-term effectiveness of the procedure (AF free survival) assessed at 1 year after the ablation is in the range of $70-82 \%$. The AF-free survival rate significantly depends on the clinical characteristics of the studied group and the presence of risk factors, especially the type of AF (paroxysmal vs. persistent), LA size and the presence of heart failure. For a safe and effective procedure the electrophysiologist should be aware of all minute details of the procedure including several tricks developed by the most experienced operators and the pre-procedural and post-procedural management recommendations. Detailed knowledge of complications of cryoballoon (CB)based ablation is mandatory. This review concentrates on the practical aspects and recommendations for a cryoballoon ablation procedure. The review is based on the authors' experience, including 800 procedures performed over 11 years with a low complication rate, and is presented within the context of the literature.
\end{abstract}

Key words: atrial fibrillation, cryoballoon, ablation, outcome, complications.

\section{Introduction}

There has been substantial progress in the diagnosis and treatment of atrial fibrillation (AF) over the last two decades. During that time catheter-based pulmonary vein (PV) isolation became the most effective method of AF treatment. However, since AF is the most common sustained arrhythmia and a significant cause of morbidity and mortality generating high costs for healthcare, there was a need for a more streamlined, more standardized procedure and with a steeper learning curve than the classic point-by-point radiofrequency (RF) catheter based PV isolation procedure. That demand was met by the development of several single-shot techniques [1]. The most successful of these was the cryoballoon (CB) ablation method, with already over half a million of pa- tients having undergone that procedure. The less commonly used single-shot tools are the 'hot balloon' and the pulmonary vein ablation catheter (PVAC). Single-shot techniques make the PV isolation procedure easier and have the potential to expand access to the interventional treatment of AF, eventually leading to a reduction of the AF-related disease burden. Clinical equivalency, in terms of clinical outcome, between CB ablation and RF ablation was documented by the 'Fire and Ice' trial and other studies [2]. In this article we will review and summarize the current knowledge regarding outcomes of cryoballoon ablation and provide a practical, 'hands-on' review of the technique on the basis of the literature and our experience with over 800 cryoballoon procedures performed during the last 11 years.

\section{Corresponding author:}

Prof. Marek Jastrzębski MD, PhD, First Department of Cardiology, Interventional Electrocardiology and Hypertension, 2 Jakubowskiego St, 30-688 Krakow, Poland, phone: +48 502545 228, e-mail: mcjastrz@cyf-kr.edu.pl

Received: 23.11.2020, accepted: 23.11.2020. 


\section{Cryo - an old new energy source}

The physical mechanism responsible for inducing freezing in tissues is based on the Joule-Thomson effect: the temperature decline of an expanding gas when it is forced to pass through a valve where it loses pressure. An important milestone in the practical application of cryoenergy was the invention of a closed cryoprobe with continuous flow of liquid nitrous oxide by New York City neurosurgeon Irving Cooper in 1961. The first cardiac application of such a cryoprobe, by John Gallagher at Duke University in the USA, was for intraoperative ablation of accessory pathways and the atrioventricular node in 1977. The introduction of steerable percutaneous cryocatheters (2001) and cryoballoons (2006) made cryoenergy available for all interventional electrophysiologists.

The aim of cryoablation is to selectively destroy the targeted tissue in a chosen area using low temperature. The main mechanisms of low temperature induced cellular damage result from a combination of direct cellular failure related to the harmful effects of ice crystal formation during hypothermia and ischemic cell death attributable to microcirculatory damage and the following vascular stasis during thawing. The current understanding of this complex mechanism divides it into the following stages: (1) freeze and thaw, (2) hemorrhage and inflammation, and (3) replacement fibrosis. In the first phase hypothermia leads to stiffening of cardiac myocytes, destruction of ion pumps and the intracellular $\mathrm{pH}$ becomes more acidic. Persistence of these changes depends on the minimal achieved temperature and the duration of cryoapplication - the shorter the exposure to hypothermic conditions, the more rapidly cells recover. This property of cryoenergy has very important clinical implications - it permit the functional assessment of the chosen ablation areas (cryomapping) without permanent cellular injury. Continuation of freezing results in formation of ice crystals (a hallmark of irreversible tissue destruction), firstly in the extracellular matrix and later intracellularly. The density of ice crystals and their dimension depend on distance to the cryocatheter, the achieved local temperature and the rate of cooling. Intracellular ice crystals cause progressive damage of intracellular organelles when extracellular crystals cause the extracellular space to become hypertonic, subsequently leading to cell shrinkage and rendering the intracellular space hyperosmotic and acidic. It finally results in damage of enzymes, protein impairment and ultimately rupture of the cell membrane. Freezing also negatively affects microcirculation which leads to (A) hyperemia, (B) interstitial edema and (C) microthrombosis, which eventually leads to tissue ischemia. After freezing is completed, the tissue slowly, passively returns to the temperature of the body - known as the thawing effect. In this phase of cryoablation, intracellular crystals are fused into larger masses, further extending the cellular dam- age. In the second phase water migrates out of the endothelial cells to re-establish the osmotic equilibrium that was interrupted earlier. This causes a further increase of intracellular osmotic pressure and can lead to further cell membrane failure. Over time a restoration of microcirculation to the previously frozen area occurs, resulting in edema and further ischemic necrosis. In the last stage, which occurs weeks after the procedure, the final lesion is created due to fibrosis and apoptosis of cells close to the borders of frozen tissue.

Size of final lesions is related to minimum achieved temperature, probe size and contact area with target tissue, duration of application(s), number of repeated freeze/thaw cycles, rate of freezing, contact pressure and the local blood flow. The safety profile of this technique is well documented. The unique feature of cryoenergy-based ablation is cryomapping: in the temperature range of 0 to $-30^{\circ} \mathrm{C}$ conduction in the cardiac tissues reversibly slows down and is eventually blocked while permanent lesions are created when the temperatures drops below -60 to $-80^{\circ} \mathrm{C}$. In contrast to the RF energy, the cryothermal energy application results in discrete homogeneous lesions that are clearly demarcated, with compact areas of fibrotic tissue, and a preserved tissue matrix with alive endothelial cell layers.

\section{Cryoballoon technology}

The Arctic Front system (Medtronic, Minneapolis, MN, USA) consists of the CryoConsole that provides the coolant and the electronic control systems, a $15 \mathrm{~F}$ steerable sheath (FlexCath) and the 10.5-F balloon catheters available in two sizes $(23 \mathrm{~mm}$ and $28 \mathrm{~mm}$ ). The first generation of cryoballoon (CB1) was introduced in Europe in 2006 and in the USA in 2011. The CB1 had four cooling jets with a distance of $7 \mathrm{~mm}$ to the balloon tip, resulting in a limited equatorial freezing region that might result in an ineffective circular lesion around the PVs. The second-generation cryoballoon (CB2), approved by the FDA in 2012, was produced on a similar platform as its predecessor, but with a shorter distance from the refrigerating jets to the balloon tip $(2.5 \mathrm{~mm})$, and the number of refrigerant jet ports has been doubled. With these improvements, the CB-2 can create a wider and more homogeneous lesion, extending from the equator up to the distal tip. These modifications led to better shortterm and long-term success rates in terms of freedom from AF. A central lumen present in these balloon catheters permits introduction of a guidewire for positioning or introduction of a special circular diagnostic mapping catheter for monitoring of $\mathrm{PV}$ potentials and positioning as well as the injection of contrast. To facilitate PV potential recording, the third generation cryoballoon (CB-3) was released in 2015 with a $40 \%$ shorter tip compared to its predecessor - but because of technical issues with the temperature probe and some dislodgement issues it 
was withdrawn from the market. The above-mentioned issues were solved and the fourth generation CB (CB-4) was released in October 2018.

A competing cryoballoon system, the POLAR X (Boston Scientific, Marlborough, MA, USA), was recently released. It was very similar in design and working principles to the Arctic Front system although minor improvements/ modifications were claimed: 1) a more uniform pressure inside the cryoballoon during inflation and the ablation phase potentially protecting from dislodgment/pop-out caused by the balloon expansion, 2) a tighter bend radius $\left(155^{\circ}\right)$ of the steerable sheath that can improve cryoballoon positioning and access to the inferior pulmonary veins, 3 ) more versatile cryoconsole that allows optional phrenic nerve and esophageal temperature monitoring, automation of patient data collection and retrieval and ability to program customized ablation quality indicators and timers, and 4) the cryoconsole allows single operator technique since the physician handling the cryoballoon can inflate, ablate, mark isolation, and stop therapy by using a foot pedal. However, the larger outer sheath diameter of the POLAR X than the ArcticFront (15.9 vs. $15 \mathrm{Fr}$ ) is potentially disadvantageous.

\section{Pre-procedural considerations and patient management}

\section{Indications}

European Guidelines 2020 concerning management of patients with atrial fibrillation recommend performing $\mathrm{PVI}$ in patients with symptoms after ineffectiveness or intolerance of pharmacological treatment with at least one antiarrhythmic drug from class I or III. This is a class I recommendation for patients with paroxysmal and persistent AF regardless of the presence of major risk factors for recurrence of AF. These guidelines also recommend performing PV isolation already after failed $\beta$-blocker treatment (IIa) or as a first-step treatment for patients with paroxysmal (Ila) and persistent (Ilb) AF, considering in the decision process the patient's preferences, potential benefits, and risks. Importantly, PV isolation is recommended as a first-line therapy to reverse LV dysfunction in AF patients when tachycardia-induced cardiomyopathy is highly probable (class I) and also as a strategy to avoid pacemaker implantation (IIa) in patients with AF-related bradycardia or symptomatic pre-automaticity pause after AF conversion (i.e. sick sinus syndrome).

The main goals and benefits of PV isolation are the reduction of arrhythmia-related symptoms and improvement in quality of life. Among asymptomatic patients PV isolation could by conducted after detailed discussion and consideration of the pros and cons, as there are no randomized trials to ensure mortality benefits in AF patients without heart failure. However, pathophysiological reasoning and some studies point to decreased mortality, stroke and heart failure rates after AF ablation [3-5].

\section{Risk of AF recurrence after ablation}

To facilitate patient counselling and selection for $C B$ ablation several risk scales that assess the risk of $A F$ recurrence after CB ablation were proposed [6]. The major risk factors connected with $A F$ recurrence include persistent AF type, obesity, enlargement of the left atrium and reduced ejection fraction of the left ventricle. These risk scores have some limitations. Firstly, they are difficult to use in clinical practice because of a lot of included components, sometimes based on hard to obtain data. Secondly, application of these risk scores in different populations provided divergent results, probably due to the inclusion of a number of risk factors that were not universal but population/operator/center dependent. To address these limitations our center developed a simplified tool based on only two universally validated, easily available and strong risk factors: AF type and left atrial (LA) size > $45 \mathrm{~mm}$ - named the "0-1-2 PL score". This score had higher predictive power, as assessed by area under the curve, than other much more complicated scores that were validated on non-Polish populations (in press).

\section{Anticoagulation}

Patients with risk factors for stroke should take adequate anticoagulation for at least 3 weeks directly before the procedure (class I) - alternatively, transesophageal echocardiography could be performed to exclude the presence of thrombi in the LA (IIa). The 2020 ESC guidelines recommend not interrupting the oral anticoagulant therapy before ablation - as such a strategy was used in trials that compared warfarin with novel anticoagulants. However, other recent guidelines (2017 HRS/EHRA Calkins et al.) considered that it is reasonable to hold one to two doses of novel oral anticoagulant prior to AF ablation with re-initiation after ablation. We believe that it is unreasonable to perform ablation on an active oral anticoagulant without immediate access to a reversible agent and that the local anticoagulant strategy should be monitored with local outcomes/complication rather than blindly rely on data from trials performed in different centers/ populations. In our center we follow the strategy of postponing the morning dose of any oral anticoagulant, with the re-initiation on the same day, $6 \mathrm{~h}$ after the vascular sheaths are removed/hemostasis achieved. No periprocedural strokes were observed in our center over several years when such a strategy of minimal interruption was followed.

\section{The cryoballoon ablation procedure}

\section{Access}

Femoral access is obtained in a typical fashion. To minimize the local trauma and the risk of arterial puncture we probe for the femoral vein by aspiration during injection of local anesthesia via a thin needle (outer di- 
ameter $0.8 \mathrm{~mm}$ ). This method can precisely pinpoint the location and depth of the femoral vein as the thin needle does not obliterate the vein lumen when puncturing the vein wall. In difficult cases ultrasound is used to visualize the femoral vein and Valsalva maneuvers to increase the size of the vein. We perform three venous punctures: one for the transseptal sheath and two to introduce the His catheter and steerable coronary sinus (CS) diagnostic multipolar catheter. Diagnostic catheters facilitate transseptal puncture by providing anatomical landmarks and are subsequently used for pacing maneuvers to confirm PV isolation, for ventricular pacing during bradycardia and for phrenic nerve pacing.

Transseptal puncture is performed using the electrophysiological approach, SL-1 sheath and the Brockenbrough (BRK-1) needle. The whole assembly/needle tip should be oriented medially and posteriorly. The orientation of the transseptal needle is adjusted according to the coronary sinus catheter/mitral annulus position by keeping the needle indicator pointing at the 4 o'clock (usually) to even at the 8 o'clock direction (in severely rotated hearts). For this the right anterior oblique view can be used as it helps to locate the dilator tip posterior to the site of the His catheter and posterior and parallel to the CS catheter. In the left anterior oblique (LAO) view the tip of the dilator should be well above the CS catheter. Contrast injection (the septal staining technique) is occasionally used to visualize the interatrial septum. In difficult cases, the interatrial septum should be visualized with intracardiac or transesophageal echocardiography to increase the safety of the puncture - guided then by the tenting sign. For fossa ovalis localization the whole assembly is slowly withdrawn under fluoroscopy in the $\mathrm{LAO} 30^{\circ}$ projection until two jumps of the catheter tip are observed, one from the superior vena cava to the right atrium (often very minimal in LAO) and the other (usually very obvious) into the fossa ovalis. We do not follow the recommendations for low fossa ovalis puncture as in our experience it makes access to the PV more difficult. The interatrial septum is then punctured by advancing the needle. At this point, the blood is withdrawn for initial confirmation of LA access. We do not use pressure monitors but rely on fluoroscopic visualization of contrast injected through the needle to confirm access to the LA. In case of doubt/poor visibility, coronary angioplasty or another 0.014" guidewire can be introduced through the needle and positioned in one of the PVs to confirm that the appropriate structure was accessed. The introducer together with the SL-1 sheath is then inserted over the needle into the LA. After this the SL-1 sheath is immediately replaced with a stiff 0.035 " guide wire, positioned in one of the left-sided PVs. A heparin bolus of 10000 $16000 \mathrm{U}$ is administered, aiming for an activated clotting time of 350-400 s; this should be performed immediately after puncture or preferably before puncture [7].

\section{Sedation and analgesia}

Shortly before the procedure intravenous acetaminophen (1000 mg) is administered to reduce the need for opioids. During the procedure, after the transseptal puncture, intravenous boluses of midazolam $(2.5 \mathrm{mg}$ ) and fentanyl $(0.05 \mathrm{mg}$ ) are used for pain/anxiety control. The procedure is usually well tolerated.

\section{Engagement and occlusion of the veins}

Engagement of the left-sided PVs is performed in LAO $30-40^{\circ}$ while right-sided PVs are engaged and ablated in the postero-anterior view and occasionally in the shallow RAO view. We use the dedicated intraluminal circular mapping catheter (Achieve catheter) to intubate all PVs starting with the left superior or the left inferior vein. The inferior PVs are located posterior and inferior to the superior PVs; therefore, once a superior or an inferior vein is intubated this directs the rotation and flexing of the Flexcath sheath to intubate the other. To prevent LA trauma/perforation by the stiff tip of the CB catheter or the Flexcath sheath the soft-tipped mapping catheter should always be exposed to lead the CB catheter. For de novo PV isolation procedure we do not recommend any preprocedural visualization of the LA anatomy or intraprocedural visualization of the veins using angiography as this is very rarely useful and has unproven outcome benefits. In the vast majority of cases veins have a typical anatomical position and can be accessed using simple maneuvers. Moreover, PVs are visualized anyway during contrast injection for determination of the balloon occlusion grade. In difficult cases, when one of the veins cannot be intubated or there is a need to confirm the presence of common trunk or an additional vein is suspected, the LA angiography can be performed by a fast injection of $50 \mathrm{ml}$ of contrast into the LA during fast ventricular pacing (250 bpm; to slow atrial emptying) - Figure 1.

Once the mapping catheter is inside the target vein, the markers on the cryoballoon body are used to position it outside of the Flexcath sheath (the second white band indicates that the cryoballoon is out of the sheath). Balloon inflation should be performed outside of the vein - in the PV antrum or LA. An antral level of isolation likely reduces collateral damage by maximizing the distance between the balloon and the phrenic nerve, the esophagus and the lungs. It is extremely important to provide adequate forward pressure to the balloon/sheath to ensure the best possible contact of the balloon with the PV antrum. Often appropriate Flexcath rotation/flexion or some 'tricks' (see below) are necessary for this. Good contact ensures rapid isolation and probably prevents re-conduction. Initial confirmation of a good contact relies on the assessment of the occlusion grade with contrast injection via the balloon catheter lumen into the occluded vein. There should be no contrast leak into the LA or a very minimal leak that will close once the balloon slightly expands during freezing. 
It might be tempting to position the balloon deeper, not in the antrum but in the proximal part of the PV, as this provides a more stable position, a perfect occlusion and a fast isolation. However, it is a very dangerous approach and cannot be pursued, and once such a position is identified, the balloon should be immediately deflated and repositioned. In large diameter superior veins it is especially easy to inadvertently position the balloon too deep. To ensure an antral position the 'proximal seal' technique should be routinely used (Figure 2). This technique is based on withdrawing the cryoballoon slightly to create a temporary contrast leak, as this will either visualize the PV ostium or will visualize the more proximal part of the vein, indicating that that the balloon, after withdrawal, was still inside the vein. In such cases a popular trick that increase the balloon diameter and stiffness and prevents it from jumping inside the PV during forward pressure is to initiate the ablation/freeze a few seconds before advancing the balloon.

For good vein occlusion and balloon contact with the antrum the proper alignment of the axes of the mapping catheter, the Flexcath sheath and the PV antrum is probably the decisive factor. Therefore, if a good occlusion cannot be initially achieved, then the vein should be intubated several times as different branches are then often engaged with the mapping catheter and the orientation of the Flexcath sheath, with regard to the antrum, as well as the sheath and catheter rotation/flexion changes. Although most of the support for the balloon is provided by the Flexcath sheath, the mapping catheter often also provides a desirable stabilization - but only when positioned deep inside the vein. Unfortunately, this prevents real-time monitoring of the $\mathrm{PV}$ isolation process, as in a deep position the catheter does not record PV potentials. Therefore, the deep mapping catheter position facilitating stabilization versus the shallow position facilitating PV potential recording should be balanced. We initially aim for recording of PV potentials, as this provides the ultimate information that the balloon was in good circumferential contact with the tissue and also because such technique facilitates avoidance of a too deep position of the balloon. Therefore, once the occlusion is achieved the circular mapping catheter should be withdrawn and rotated ('back-flipped') so that it rests on the balloon and records PV potentials - Figure 3. Only when this repeatedly results in instability/leak/balloon displacement should a deeper positioning of the mapping catheter be allowed. In such cases instead of the real-time PV potentials monitoring, other methods for confirmation of PV isolation should be used (see below).

\section{Assessment of the freeze quality and freeze dosing}

Assessment of proper positioning of the balloon continues during the freeze application by processing of the

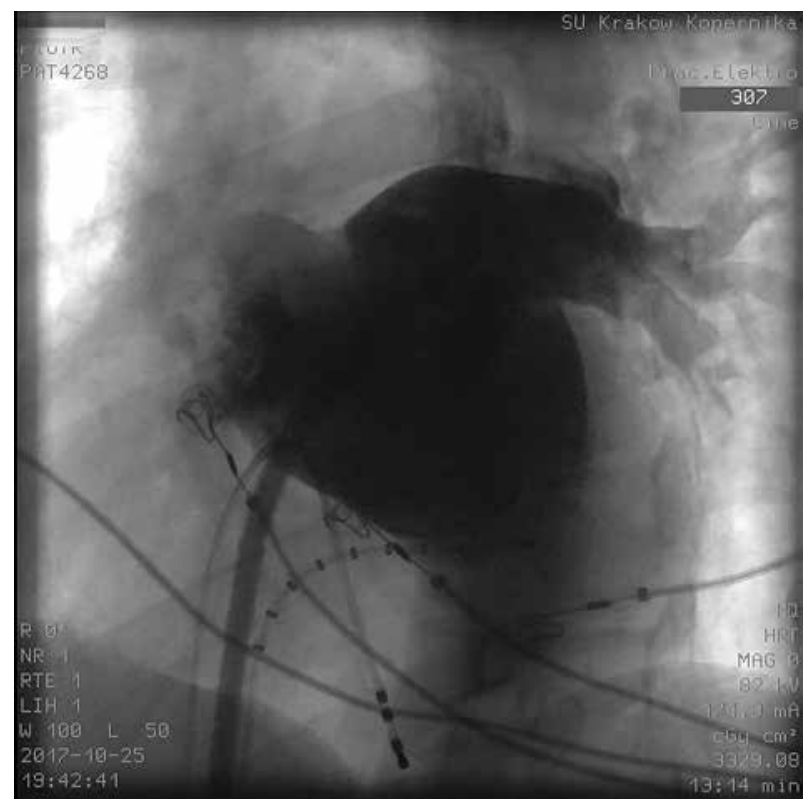

Figure 1. Left inferior pulmonary vein could not be localized and the angiography of left atrium was performed with $50 \mathrm{ml}$ of contrast injected via the Flexcath sheath during fast ventricular pacing (to slow atrial empting); this visualized left common trunk and confirmed absence of a separate inferior pulmonary vein

real time data from the cryoconsole (temperature drop) and the circular catheter (disappearance of PV potentials, "time to isolation"). Too fast temperature drop (colder than $-40^{\circ} \mathrm{C}$ at $30 \mathrm{~s}$ ) and too deep nadir temperature (colder than $-56^{\circ} \mathrm{C}$ at any time) indicates a dangerously deep position of the balloon and the freeze should be aborted. As a rule, in such cases a more proximal position with proper temperature behavior can be obtained. Too slow temperature drop resulting in too high temperature at 1 min (warmer than $-35^{\circ} \mathrm{C}$ ) should also prompt abortion of the freeze as the balloon is probably in poor contact with the antrum. The thaw time also indicates good vs. poor contact, and shallow vs. deep balloon position. These phenomena and the proper temperature behavior pattern are illustrated in Figure 4.

Short time to isolation is considered the best predictor of a durable lesion and another indicator of a proper balloon position. Time to isolation below $<40 \mathrm{~s}$ is a very good sign, and likely a single, short (120-150 s) freeze per such vein might be enough. Time to isolation $<60 \mathrm{~s}$ is probably acceptable, while a longer time to isolation or lack of isolation at the end of cryoapplication requires a second freeze - after some change of the balloon position/alignment. The thaw time/rate also informs about the balloon position within the antrum/vein (Figure 4) and is a marker of a durable lesion. However, most likely none of the predictors is good enough to allow the operator to be absolutely certain than the vein was permanently 

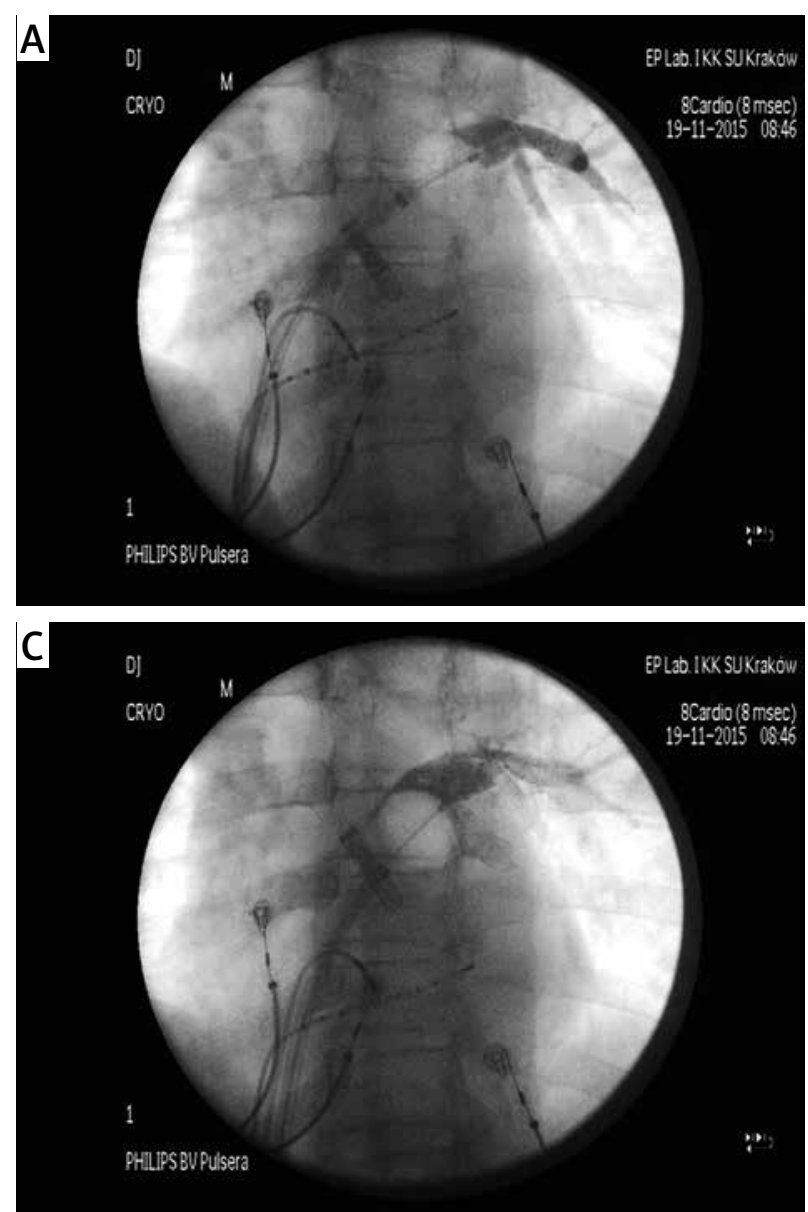

isolated; therefore, other factors should be considered for individualized cryoenergy dosing. Recommended freeze time per vein and per application evolved over the years. Despite multiple studies comparing various fixed and titrated dosing protocols, this part of the procedure is still far from evidence-based standardization. In 2009 we used 3 freezes of 4-5 min duration per vein; nowadays, often a single 120-180 s application is considered enough. Our standard protocol is a single 240-second freeze per vein for left-sided PVs and a single 180-second application per vein for right-sided PVs. These times are often modified and additional freezes are often added according to the time to isolation, achieved temperature drop, phrenic nerve behavior and the perceived arrhythmogenic potential of a particular vein - Figure 5. When dozing the freeze time, several simple rules should be considered. 1). Fast temperature drop and a quick isolation is not equivalent to slow temperature drop and late vein isolation, even if similar temperatures are reached. Slow temperature drop probably indicates a small area of balloon contact with the antrum and/or constant rewarming and the lesion might not be permanent. 2). Increasing freeze time in cases with slow temperature drop will increase the risk of collateral damage, as with the increased duration of the freeze the depth of ice penetration into the nearby tissues grows. 3). Bonus freeze is usually not necessary

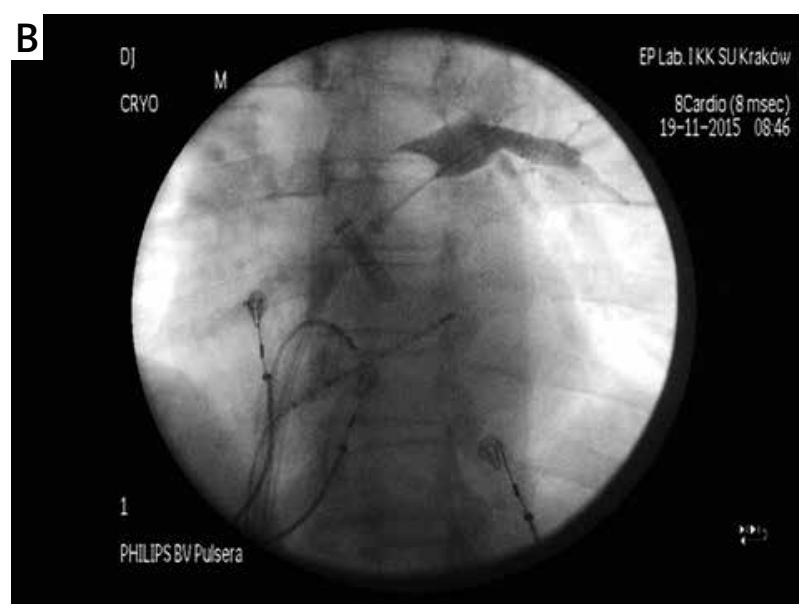

Figure 2. Proximal seal technique. A - A good vein occlusion indicated by the contrast retention during an apparently proximal balloon position. However, instead of initiating the freeze application, the balloon was slightly pulled back to create a temporary contrast leak. B - During balloon withdrawal instead of a contrast leak to the left atrium - a more proximal part of the left superior pulmonary vein was visualized indicating that the balloon was almost completely inside the vein. $\mathbf{C}$ - The balloon was pulled back more until the created contrast leak delineated the antrum and indicted the proper proximal position of the balloon

when the initial freeze had very good parameters (time to isolation < $40 \mathrm{~s}$ and the temperature at $1 \mathrm{~min}$ below $\left.\left.-40^{\circ} \mathrm{C}\right), 4\right)$. Evidently poor freeze parameters: time to isolation $>90 \mathrm{~s}$ or temperature at $1 \mathrm{~min}$ warmer than $35^{\circ} \mathrm{C}$ indicate the need for another freeze. 5). Shorter freeze time and no-bonus freeze policy decreases left atrial dwell time, while too short freeze time ( $<180 \mathrm{~s}$ or even below $240 \mathrm{~s}$ for left sided veins) is related to a smaller lesion and a higher risk of re-conduction.

\section{Additional positioning techniques}

Several 'tricks' have been proposed to improve the acute results of the cryoballoon based PVI. The most common trick used to facilitate the occlusion of the inferior PVs is the hockey stick technique - for this the Flexcath sheath should be maximally bent and pushed up to provide support for the balloon from the superior position in the left atrium (Figures 3 and 6); the mapping catheter/guidewire should be placed in the most inferior (caudal) branch of the PV, resulting in a hockey stick figure on the fluoroscopy. If despite the hockey stick technique there is still a poor occlusion/temperature or the vein is not isolated at 40-60 s the pull-down maneuver can be used. It is based on the assumption that the superior part of the balloon, frozen to the superior aspect of the inferior vein, stabilizes the cathe- 


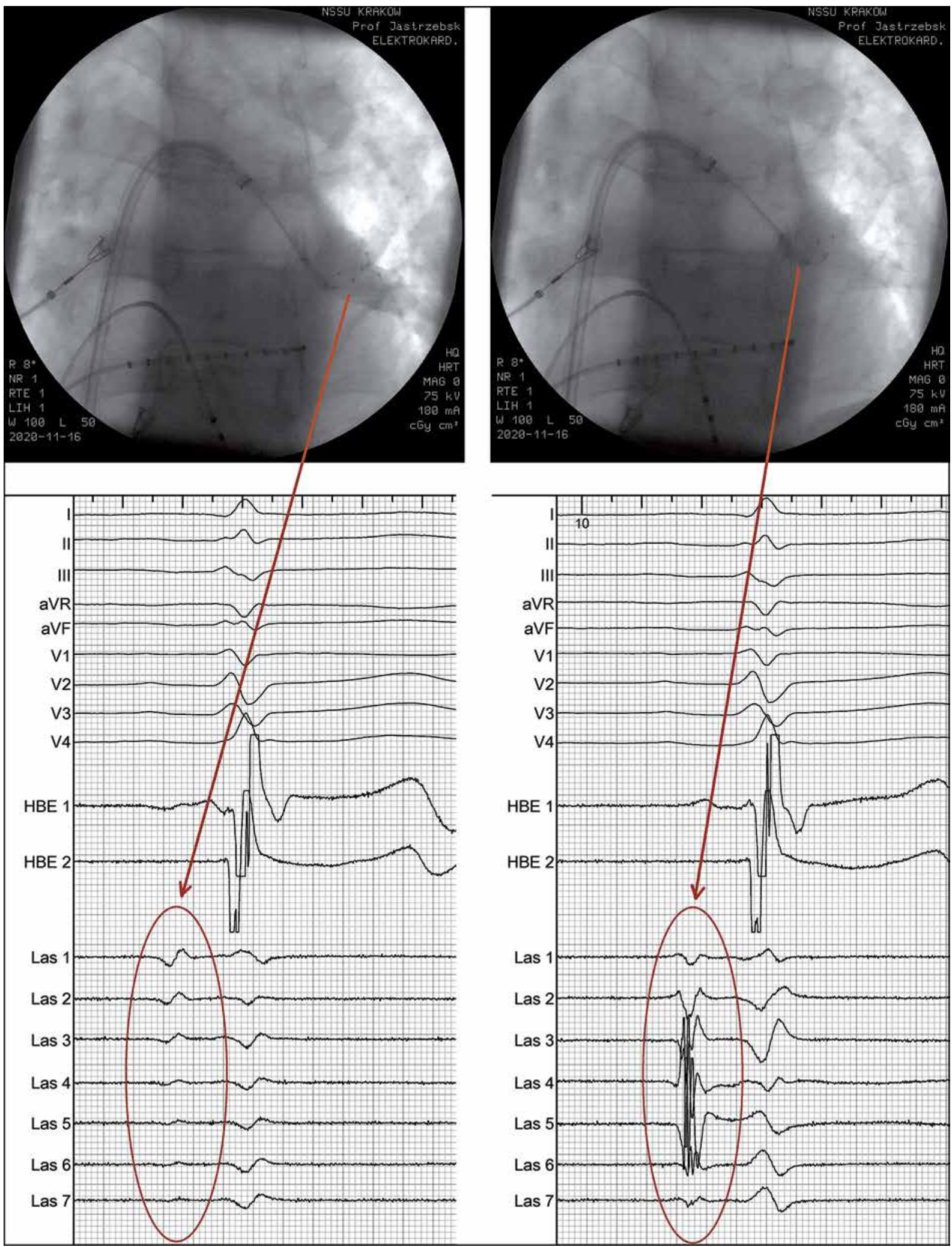

Figure 3. Left inferior pulmonary vein is occluded with the cryoballoon. Distal position of the circular mapping catheter results in lack of pulmonary vein potentials (circle, left panels). After rotation with pulling of the circular mapping catheter it backflips and rests on the balloon where ample pulmonary vein potentials are recorded (right panels) 

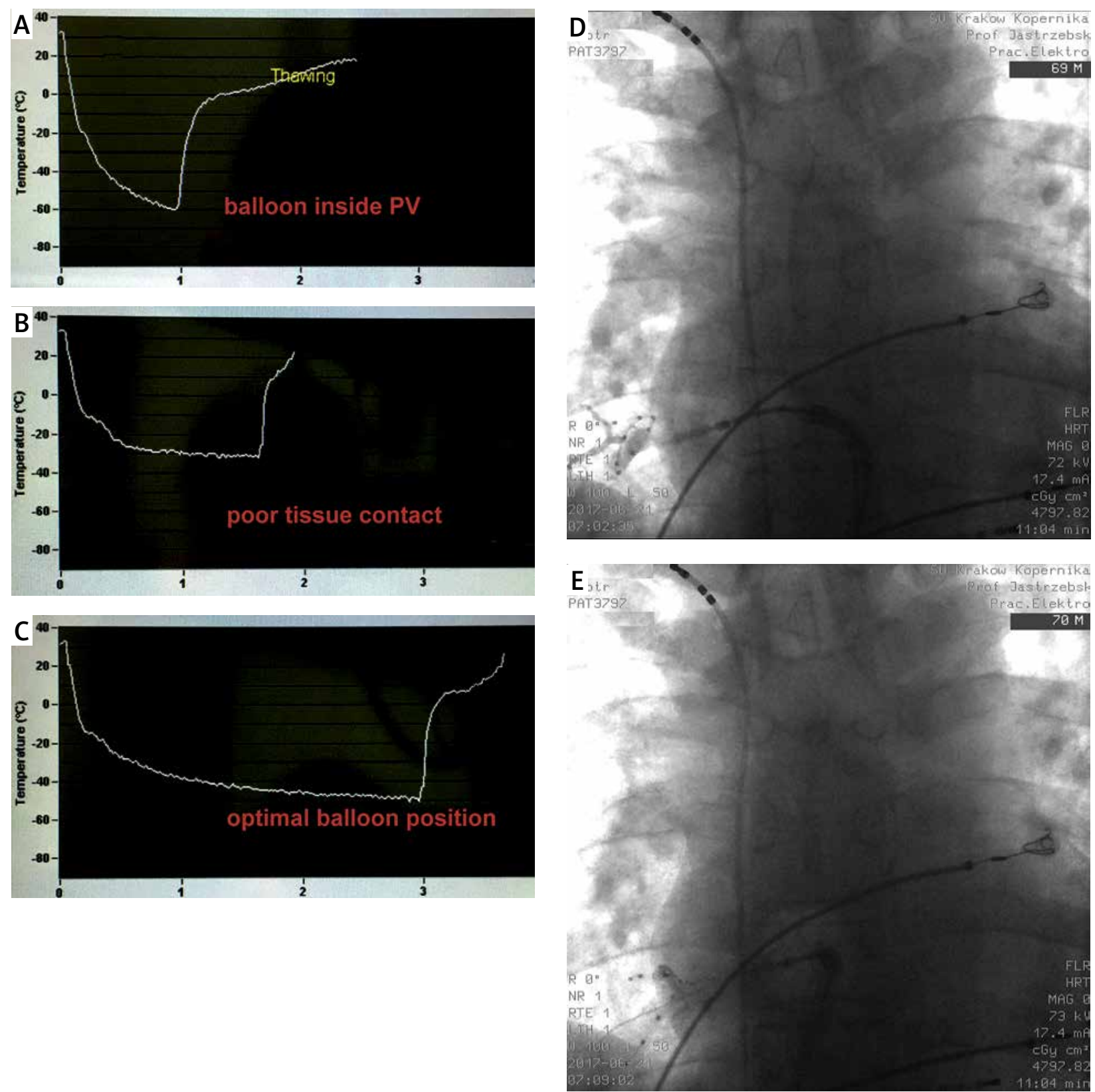

Figure 4. Temperature behaviour patterns observed in the same patient during three separate freeze applications in the right inferior pulmonary vein. The first freeze (A) illustrates rapid temperature drop exceeding $-40^{\circ} \mathrm{C}$ at $30 \mathrm{~s}$. This had prompted to stop the application at $1 \mathrm{~min}$ when the temperature dropped to $-60^{\circ} \mathrm{C}$. Such rapid temperature drop was caused by positioning the balloon inside the pulmonary vein (D). Note very slow re-warming resulting in a long thaw time. After the balloon was repositioned to a more proximal position (E), the next freeze resulted in a very sluggish temperature drop that plateaued around $-30^{\circ} \mathrm{C}(\mathrm{B})$. That indicated a poor tissue contact and prompted to stop the freeze to prevent local oedema/temporary lesion; that freeze should have been stopped earlier - already at $1 \mathrm{~min}$. The third freeze was performed with the balloon in the same proximal/antral position as before $(\mathrm{E})$, albeit with a more forward pressure and strong counterclockwise rotation of the Flexcath sheath; this resulted in the optimal balloon position and a good tissue contact - as indicated by the proper temperature behaviour; the freeze was completed after 3 min 


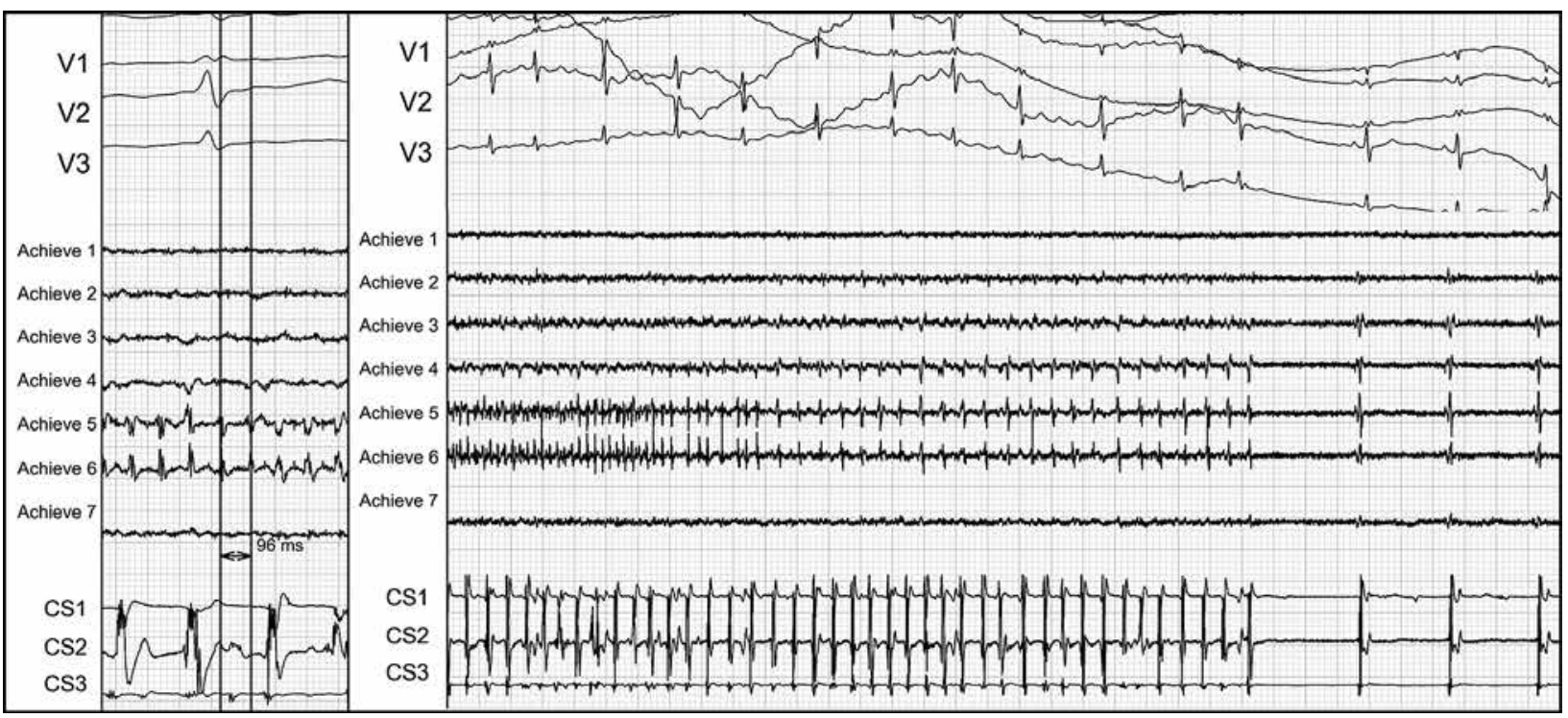

Figure 5. Electrical activity in the left superior pulmonary vein very suggestive that this vein was the culprit vein for paroxysms of atrial fibrillation. Left panel: the spontaneous activity in that vein was much faster than in the rest of the left atrium: cycle of 90-100 ms versus 250 ms in the coronary sinus (CS). Right panel: during freeze application the spontaneous activity in the vein slowed down and the sinus rhythm was restored. In our opinion such a vein deserves a bonus freeze regardless if the initial freeze resulted in a fast isolation or not
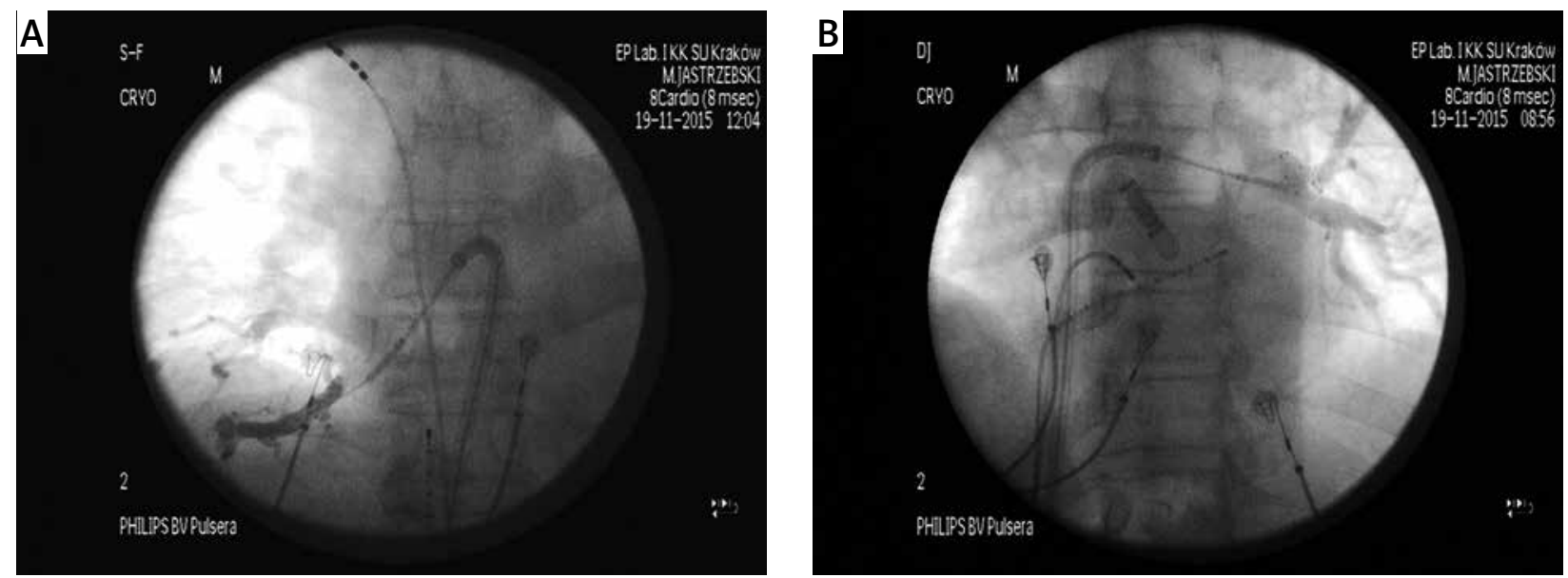

Figure 6 . When direct approach to the occlusion of the inferior pulmonary veins fails, the hockey stick technique should be tried. This relies on the strong flexing of the Flexcath sheath to provide support for the balloon from the superior position; it is advised to intubate the most inferior (caudal) branch of the pulmonary vein to facilitate this approach. The resulting hockey stick figure on the fluoroscopy can be appreciated on the upper panel in case of an inferior pulmonary vein occlusion and in the lower panel in a case of a superior pulmonary vein occlusion

ter and pulling the catheter will occlude the vein by closing the inferior gap. This maneuver is very effective in the acute setting. Often the isolation occurs within a few seconds after pulling the catheter and the temperature curve will show an additional small but abrupt decrease. However, the usefulness of the pull-down technique is debatable. Some argue that after 40-60 s the balloon is already covered with an ice layer and cannot provide a good tissue contact at the gap; consequently the isolation will be temporary and the lesion not transmural. We do not share that opinion. The pulldown maneuver has several beneficial effects: 1) it increases the contact area of the cryoballoon at its inferior perimeter, 2) increases contact pressure on local tissues which decreases warming effects of local blood flow, 3) closes the gap preventing warming effects of PV blood flow. These mechanisms profoundly impact heat transmission (subtraction) and lesion size. We are not aware of any studies showing worse outcomes in patients treated with the pull-down technique or supporting the 

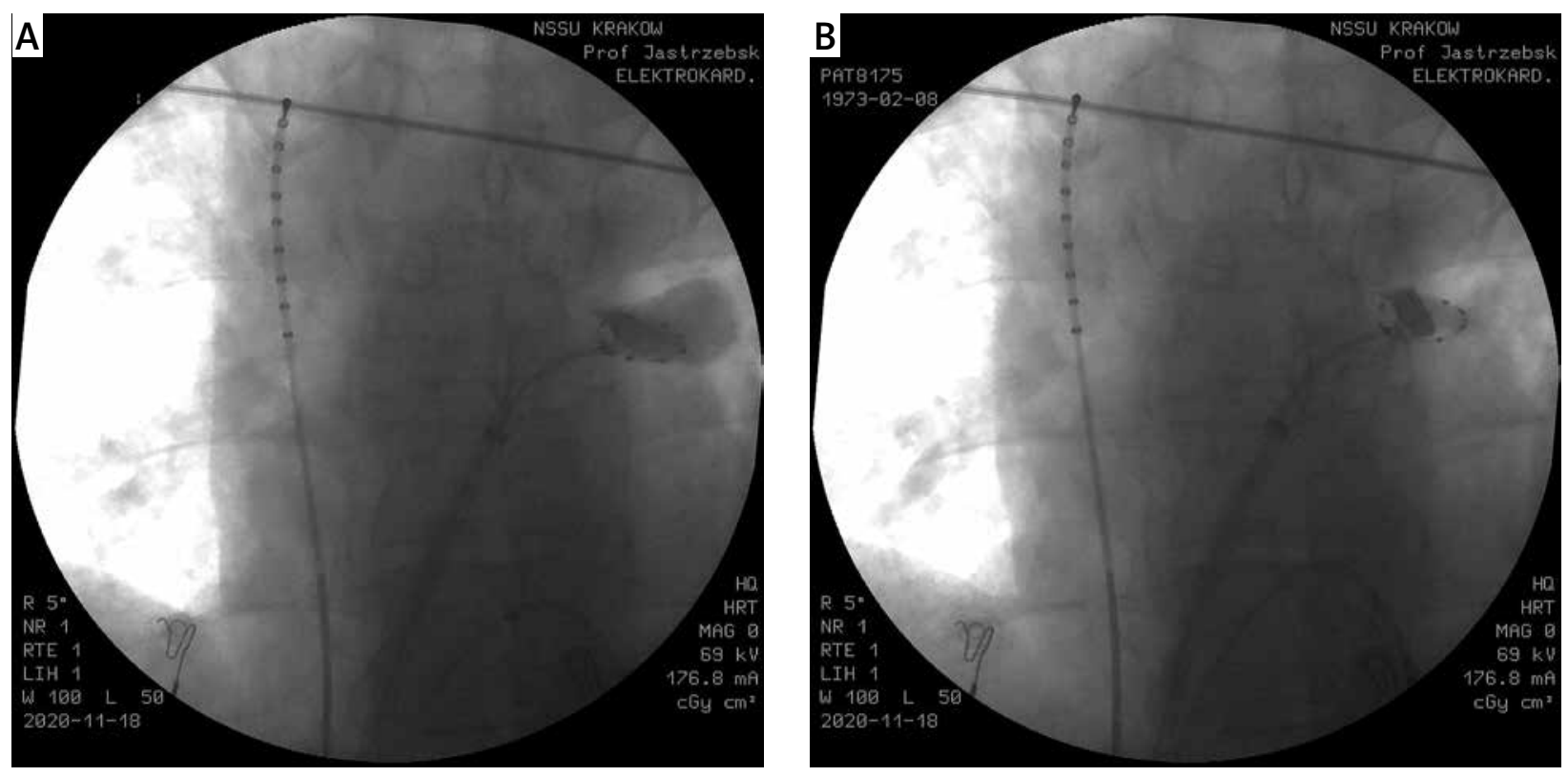

Figure 7. Despite automatic deflation of the balloon by the cryoconsole and rewarming to $36^{\circ} \mathrm{C}$ and, the catheter should not be repositioned because it is still frozen to the pulmonary vein - as evidenced by the residual ice plug in the proximal part of the vein. In the left panel the ice completely occludes the vein as evidenced by contrast retention. On the right panel with fluoroscopic image obtained some $20 \mathrm{~s}$ later, the contrast completely leaked into the left atrium but the circular mapping catheter is still firmly frozen to the vein

speculation that the veins isolated with the pull-down technique are not permanently isolated; on the contrary, there are studies showing that the pull-down maneuver results in permanent isolation of PVs $[8,9]$. Moreover, the reasoning that an ice layer prevents cryoenergy transmission seems faulty; thermal conductivity of ice is good. The best evidence comes from the cryoablation itself - damage to the phrenic nerve or other adjacent structures occurs despite the lack of a direct contact with the balloon, low temperatures reach the phrenic nerve via other frozen tissues - composed mainly of water i.e. via an ice layer. We use the pull-down technique also in cases without contrast leak, as in such cases it can also result in sudden isolation of the vein, through the above-mentioned mechanisms no. 1 and 2. Occasionally the gap or narrow area of contact between the balloon and the PV antrum is at the superior perimeter of the balloon and instead of the pull-down, a push-up maneuver will lead to the increased contact surface area and increased tissue compression resulting in fast PV isolation. The overlapping freeze/lesion technique is an alternative and complementary technique to the pull-down/ push-up maneuvers. It is applicable especially to cases with a large diameter antrum of a common trunk that cannot be proximally occluded with the $28 \mathrm{~mm}$ balloon. The overlapping freeze applications are performed using the Flexcath sheath to obtain a firm contact of the balloon with different parts of the antral perimeter during the subsequent freezes. This technique seems inferior to the pull-down maneuver as an incomplete PV occlusion results in an unbroken blood flow in the vein and tissue warming - including at the area of balloon contact.

\section{Thawing and balloon deflation}

After completion of the desired freeze time the balloon is automatically deflated when the temperature reaches $20^{\circ} \mathrm{C}$. However, often the balloon is still frozen to the PV/antrum - as demonstrated in Figure 7. Repositioning the balloon immediately after deflation might damage the PV wall; it is recommended to wait until the ice cap melts completely. However, often it does not contain contrast and is, therefore, not visible; gentle pulling can be used to ensure that the catheter in not adhered to tissue but moves freely. Very slow thawing should raise the suspicion that the balloon was inside the PV, while very fast thawing indicates that the surface area of balloon contact with the antrum was small and perhaps a bonus freeze with slightly different position should be made even if the PV isolation was achieved. When it is planned to occlude another vein the balloon should be retracted into the sheath; for this the balloon should be first inflated and deflated while advancing the push button on the catheter handle that extends the balloon to maximum length.

\section{Electrophysiological assessment of pulmonary} vein isolation

Regardless of the energy source used for ablation, demonstration of PV isolation is obligatory. Real-time 


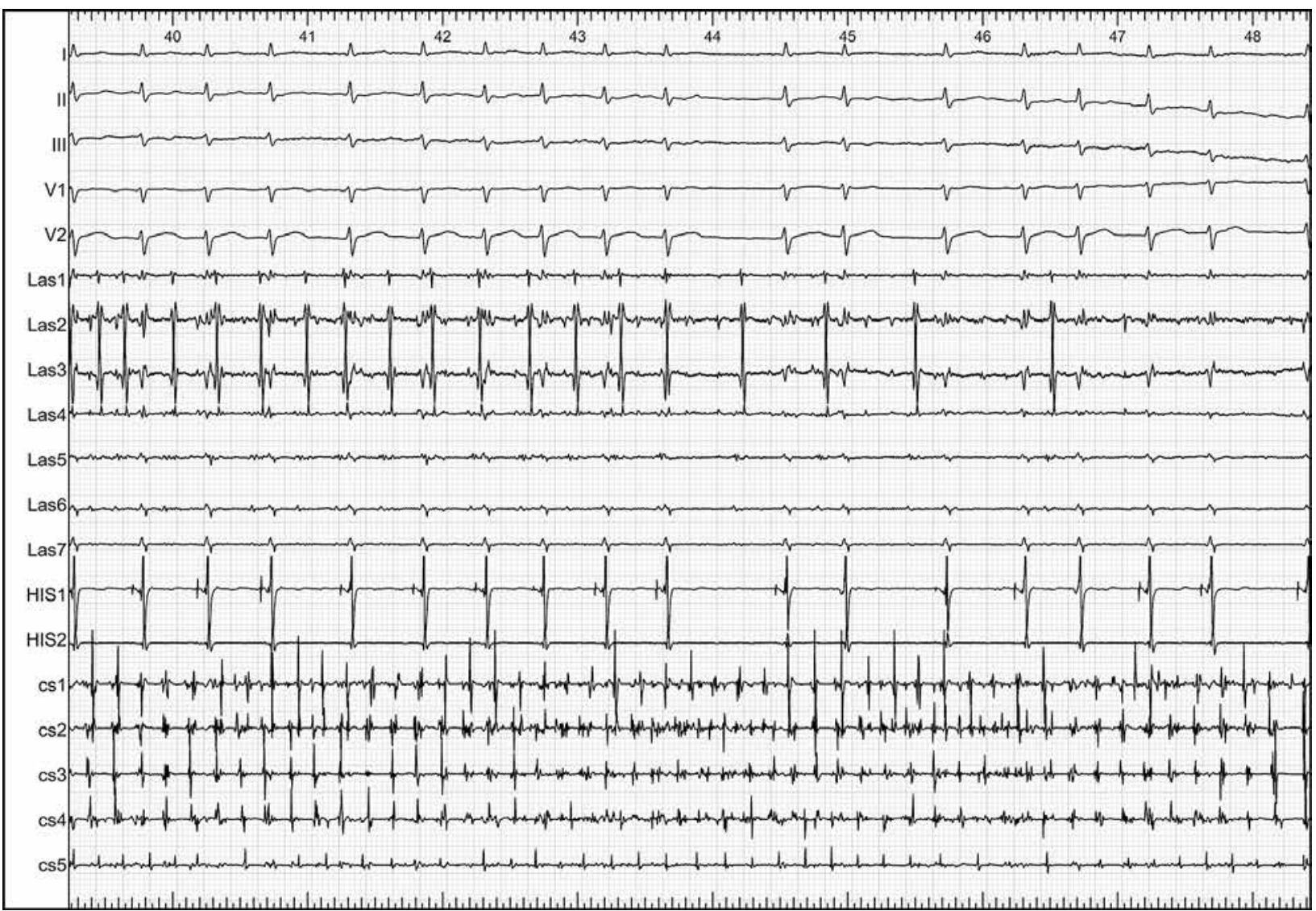

Figure 8. Cryoballoon based isolation of the left inferior pulmonary vein during a re-do procedure in a patient after remote point-by-point RF pulmonary vein isolation. Real time recording of the pulmonary vein potentials allow easy differentiation of PV potentials and far-field potentials

monitoring of PV potentials and rapid PV isolation observed in the majority of cases makes this task easy during CB ablation (Figure 8). This method leaves little doubt what the PV potential and the far-field potential are, and when block occurs, therefore, additional maneuvers are rarely necessary to confirm $\mathrm{PV}$ isolation.

However, when the real-time recording of PV potentials is not feasible, alternative methods have to be used. An elegant proof that the vein was isolated is the observation of a spontaneous PV electrical activity dissociated from the atrial activity (Figure 9). However, this is a relatively uncommon phenomenon. Constant pacing from the circular mapping catheter during freezing enables one to demonstrate the exit block as it will manifest itself with delayed activation and then loss of synchronized atrial activation while PV capture is ensured by the stable position of the frozen catheter. Pacing inside the PV with $5 \mathrm{~V}$ output after the ablation and at the site of recorded suspicious potentials also differentiates local potentials from far-field potentials that are usually not captured with moderate/low output. Alternatively, the template method can be used by obtaining a proximal recording of the PV potentials before the freeze and comparing it with the recordings from the same position obtained after the freeze. In the case of doubt, if the remaining potentials are far-field or PV potentials classic electrophysiological maneuvers should be performed using the coronary sinus catheter and the His bundle catheter for right atrial, CS and left appendage pacing for differentiation.

\section{Extended use of cryoballoon catheter for} ablation/isolation of non-PV targets

Pulmonary vein isolation is the cornerstone of the AF ablation procedure, but outcome benefits of ablation of other targets are less well documented or controversial. However, in selected patients ablation of non-PV sites might be critical to achieve a good outcome. Cryoballoon can be used (off-label) to isolate the superior vena cava and the persistent left superior vena cava using a similar approach as with pulmonary veins [10]. Extreme caution should be used in such cases as the risks of such use of the cryoballoon are not defined and vein stenosis and damage to left the phrenic nerve, the sinus node or other structures are quite possible. The left atrial appendage can also be the source of AF triggers, and CB based isolation of the left atrial appendage was reported to improve the outcome [11]. Potentially, CB based ablation might be a better option for such procedures than the point-by 


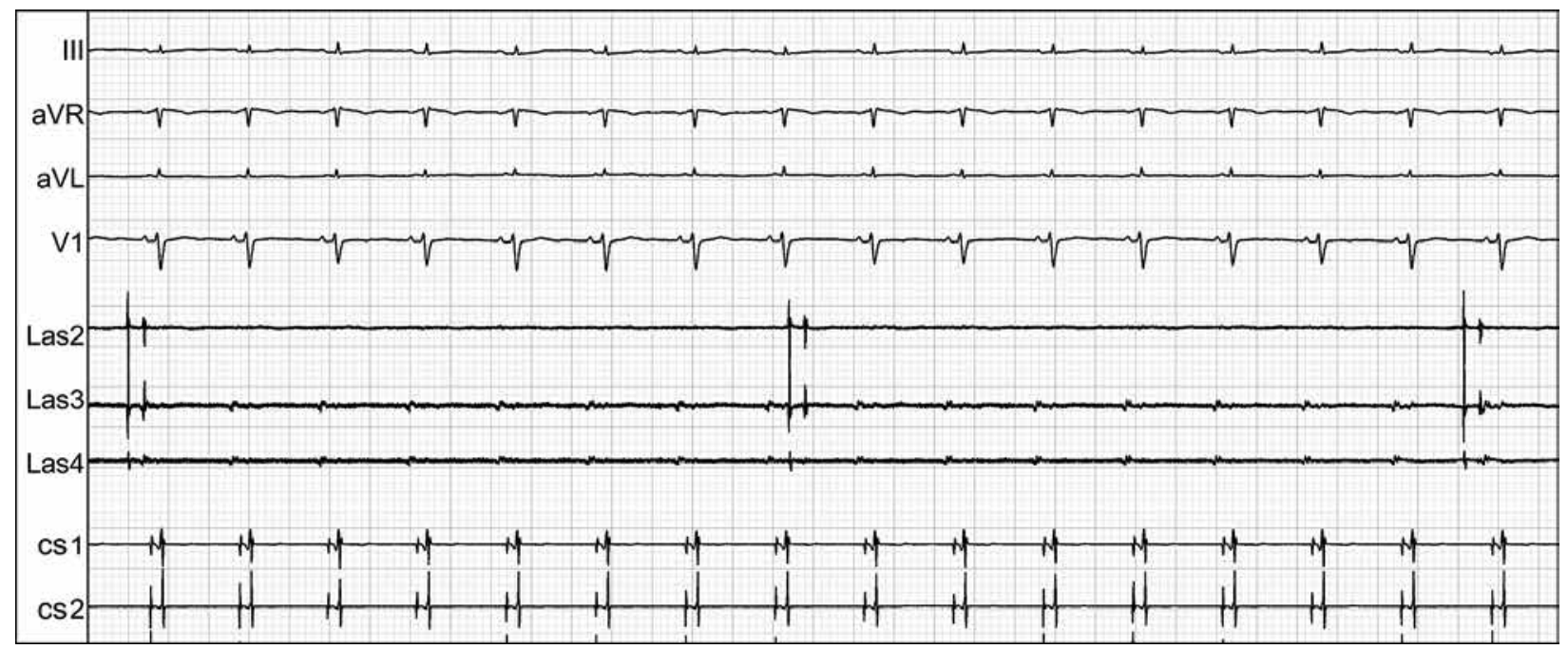

Figure 9. Electrical isolation of the left inferior pulmonary vein was diagnosed by observation of an exit block there was a spontaneous slow electrical activity in the pulmonary vein (Las2 - Las4) that was dissociated from the regular electrical atrial activity and the sinus rhythm, evident in the coronary sinus recordings (CS1-CS2) and the surface ECG leads (III, aVR, aVF, V1), respectively

point RF technique. However, undefined risk of damage to the right phrenic nerve, coronary arteries, appendage perforation and mechanical dysfunction of the isolated appendage, with related thromboembolic risk, makes this procedure controversial and justified only in highly selected cases with evident triggers in that structure. It was recently shown that the cryoballoon can be used for isolation of the posterior wall and the creation of a roof line [12]. Such a procedure is technically challenging, time consuming and potentially related to the increased risk of damage to the esophagus and creation of an atrioesophageal fistula. Until further studies characterize the outcome benefits and safety of such an approach it cannot be recommend for routine use.

\section{Post-procedural management}

Immediately after the procedure we routinely perform a short echocardiographic study, still in the electrophysiological laboratory, to exclude pericardial effusion. If there is substantial effusion, regardless of symptoms, pericardiocentesis is not deferred but immediately performed. Post-procedural groin management depends on the results of the echocardiographic examination. If there is any trace of pericardial effusion, or the transseptal puncture was troublesome, protamine is administered, the sheaths are removed and a figure-of-eight suture is used to stop the bleeding. If the result of the echocardiographic examination is normal then the sheaths are left and removed on the ward after $5 \mathrm{~h}$. Oral anticoagulation is either normally continued or if the morning dose was skipped then it is administered $5 \mathrm{~h}$ after obtaining groin hemostasis. Long-term anticoagulation is always continued for 2 months, regardless of the presence of risk factors for stroke, although probably one month should suffice as the healing process is at that time already completed. Necessity of a long-term continuation of anticoagulation should be determined on the basis of the patient's stroke risk profile and not on the apparent success or failure of the ablation procedure. Preprocedural antiarrhythmic drugs are usually continued for 3 months of the so-called blanking period. In patients with longterm persistent atrial fibrillation and/or heart failure, occasionally amiodarone is continued for 6 months to facilitate atrial remodeling. Monitoring for AF recurrence is based on symptoms, periodic ECG monitoring, Holter ECG monitoring (every 6-12 months) and occasionally by implantable ECG recorders - Figure 10 [13]. It is well documented that the success rate defined according to the guidelines-recommended detection of a 30-second long AF episode depends on the intensity of ECG monitoring - to the point when after the implantation of the continuous ECG recorder it loses its original sense and other measures of success have to be applied (AF burden rather than the 30-second AF episode detection). However, since the procedure is mainly performed for control of symptoms, extensive search for asymptomatic episodes of AF has undefined practical value.

\section{Acute and long-term outcome}

Several studies and metanalyses have assessed the acute and long-term efficacy of cryoballoon-based PVI. The reported success rate of PV isolation during the procedure is about $98 \%[14,15]$. Despite this, the long-term effectiveness of the procedure (AF free survival) assessed at one year after the ablation is in the range of $70-82 \%$. The AF-free survival rate significantly depends on the clinical characteristics of the studied group and the presence of risk factors, especially the type of AF - paroxysmal vs. 


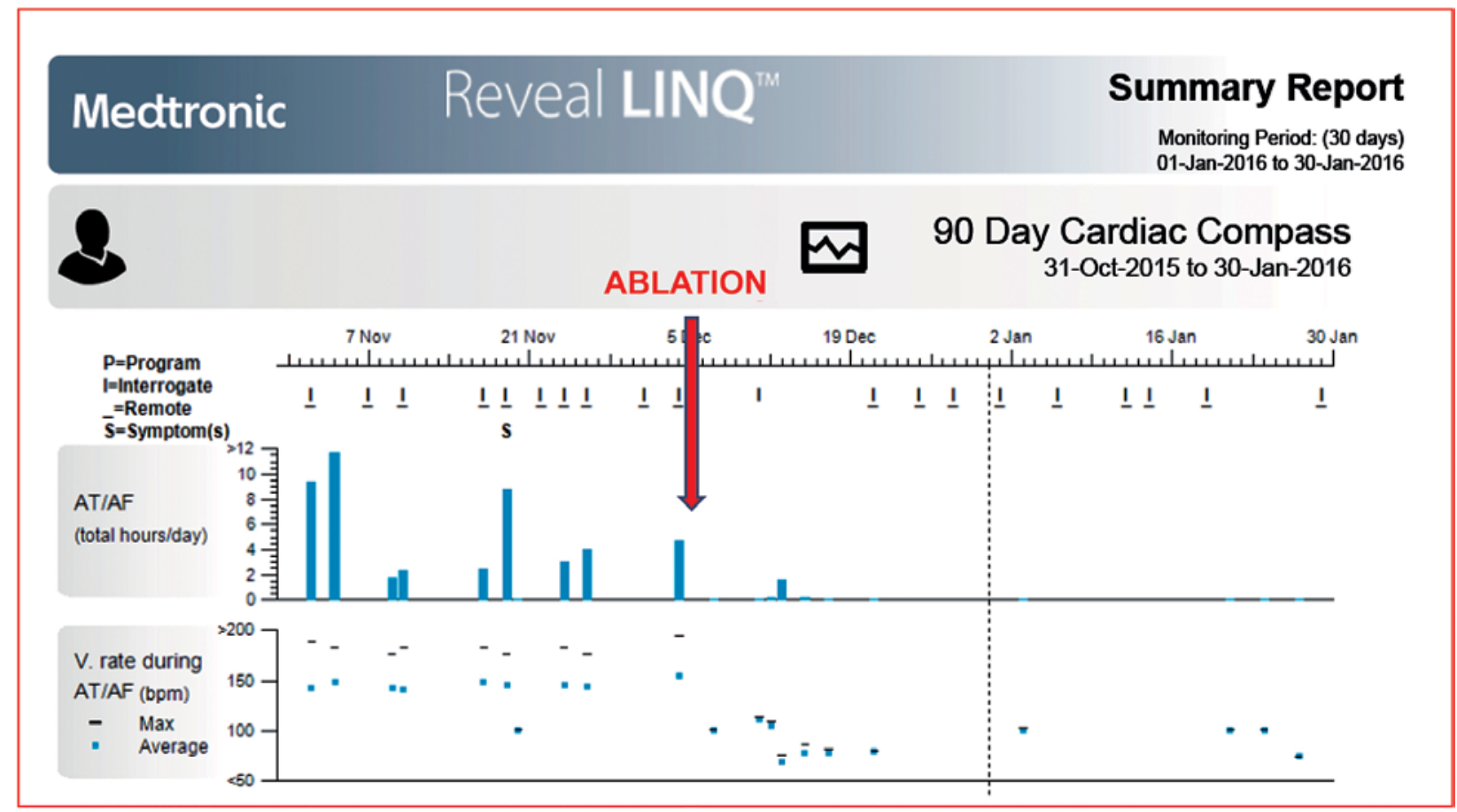

Figure 10. Implantable ECG recorder enables to monitor effect of cryoballoon ablation in a much more accurate fashion than the periodic ECG monitoring. Several AF episodes (blue bars) were confirmed before the pulmonary vein isolation (red arrow); while after the procedure there was only one AF episode - during the healing phase/blanking period. That confirmed the good result of the procedure

persistent, LA size and the presence of heart failure [15, 16]. Patients with persistent AF have a poorer long-term outcome than patients with paroxysmal AF - 55-72\% vs. $75-81 \%$ respectively $[15,17,18]$. Patients with heart failure probably have the greatest survival benefit from AF ablation (CASTLE-AF trial) despite the significantly lower AF-free survival after ablation than patients with preserved left ventricular function [19-22]. In our center we observed similar efficacy of ablation with almost $80 \%$ of patients without recurrence at 1 year. Type of AF and LA size were the strongest predictors of failure in our population [23].

Both $\mathrm{CB}$ and RF ablation techniques are considered as standard methods with similar outcomes. However, based on the largest comparison of both techniques - the "Fire and Ice study" - patients after CB ablation had significantly fewer all-cause re-hospitalizations, cardiovascular re-hospitalizations, re-ablations, and direct current cardioversions after the index procedure [2, 16, 24, 25]. In our opinion there are several important advantages of $\mathrm{CB}$ ablation making it the preferred approach. During $\mathrm{CB}$ ablation a more stable position of the catheter can be achieved, which is less sensitive for beat-to-beat heart and respiratory motions. With CB there is better control over application, which translates to more predictable, more continuous and homogeneous lesions, that are less arrhythmogenic. The rate of secondary, iatrogenic LA arrhythmias after cryoballoon ablation is markedly lower than after the point-by-point RF technique (very high for inexperienced operators, non-negligible for experienced operators). The PV re-connection rate after CB ablation is dramatically lower than after RF ablation. The most striking difference that makes the $C B$ ablation the method of choice for many operators is the speed of PV isolation; it usually takes less than 1 min from the balloon apposition in the antrum to the electrophysiologically confirmed PV isolation, and this translates into a much shorter LA dwell time, usually < 30 min. In contrast to RF ablation there is usually little doubt whether PV was isolated or not. General anesthesia during ablation is not necessary as the procedure is less painful than the RF ablation.

\section{Adverse events and prevention of phrenic nerve injury}

Prospective, registry-based data show that approximately $4-14 \%$ of patients undergoing AF catheter ablation experience complications, 2-3\% of which are serious/potentially life-threatening. The frequency of complications after CB ablation is similar, however, it differs to some extent between studies - Table I [26-30]. Based on the large clinical trials, in which mainly experienced high-volume centers take part, major complications occur in approximately $4.0 \%$ of patients; this is similar to data coming from our center. Complications occur mostly within the first $24 \mathrm{~h}$ after the ablation. The spectrum of complications includes hematoma and/or 
Table I. Frequency of major complications after cryoballoon ablation

\begin{tabular}{|c|c|c|c|c|c|c|}
\hline Parameter & $\begin{array}{c}\text { Andrade [26] } \\
2019 \\
(n=231) \\
\end{array}$ & $\begin{array}{c}\text { Hoffmann [27] } \\
2019 \\
(n=2329) \\
\end{array}$ & $\begin{array}{c}\text { Rottner [28] } \\
2018 \\
(n=563) \\
\end{array}$ & $\begin{array}{c}\text { Kuck [29] } \\
2016 \\
(n=374) \\
\end{array}$ & $\begin{array}{c}\text { Luik [30] } \\
2015 \\
(n=156) \\
\end{array}$ & $\begin{array}{c}\text { Jastrzebski [23] } \\
2020 \\
(n=589) \\
\end{array}$ \\
\hline Death & 0 & $2(0.09)$ & $1(0.18)$ & 0 & 0 & 0 \\
\hline $\begin{array}{l}\text { Pericardial effusion needed } \\
\text { treatment }\end{array}$ & $1(0.43)$ & $6(0.27)$ & 0 & $1(0.27)$ & $2(1.28)$ & $5(0.85)$ \\
\hline Pericarditis & $3(1.29)$ & $4(0.18)$ & N/D & N/D & N/D & $1(0.17)$ \\
\hline Stroke & $1(0.43)$ & $4(0.18)$ & $1(0.18)$ & $2(0.54)$ & 0 & $1(0.17)$ \\
\hline Transient ischemic attack & $2(0.86)$ & 0 & $1(0.18)$ & 0 & 0 & $1(0.17)$ \\
\hline Major groin complication & $1(0.43)$ & $10(0.9)$ & $3(0.54)$ & $7(1.89)$ & $6(3.84)$ & $9(1.53)$ \\
\hline $\begin{array}{l}\text { Persistent* phrenic nerve } \\
\text { palsy }\end{array}$ & $3(1.29)$ & $22(0.99)$ & $15(2.7)$ & $10(2.7)$ & $5(3.2)$ & $7(1.17)$ \\
\hline $\begin{array}{l}\text { Permanent phrenic nerve } \\
\text { palsy }\end{array}$ & 0 & $8(0.36)$ & $3(0.54)$ & $1(0.27)$ & 0 & $1(0.17)$ \\
\hline Atrioesophageal fistula & 0 & 0 & 0 & 0 & 0 & 0 \\
\hline Pulmonary vein stenosis & 0 & 0 & 0 & 0 & 0 & 0 \\
\hline ASD requiring intervention & N/D & $N / D$ & $N / D$ & N/D & N/D & $8(1.36)$ \\
\hline
\end{tabular}

Numbers in parentheses denote percentage; * persistent indicates present after discharge; ASD - atrial septal defect.

pseudoaneurysm at the groin access site, cardiac tamponade/pericardial effusion, thromboembolism causing cerebrovascular accidents, phrenic nerve injury/palsy, migraine attacks, silent cerebral lesions, hemoptysis, periesophageal vagal nerve injury/gastroparesis, pericarditis and iatrogenic atrial septal defect.

The periprocedural death rate related to $\mathrm{AF}$ ablation is approximately $0.2-0.98 \%$; however, in the majority of CB studies and in our practice no deaths were observed [31]. The most common (1-0.5\%) life-threatening complication is tamponade [32]. Pulmonary vein stenosis and atrioesophageal fistula formation are complications occurring mainly after RF ablation, hardly ever after CB ablation [33]. We believe that monitoring temperature in the esophagus, recommended by some to prevent atrioesophageal fistula formation, is not justified. Frequency of this complications after CB based ablation (1:10 000) is 10 times lower than after RF ablation (1:1000), and likely mostly related to positioning the CB deep within the left inferior PV [34]. Routine prescription of proton pump inhibitors to facilitate healing of esophageal injury/prevent fistula formation, recommended by some, has unproven value. The practice of stopping the freeze when the temperature drops below $-56^{\circ} \mathrm{C}$, limiting the time and number of freezes and following the proximal seal technique probably further reduces this complication.

The complication most feared by the patients is stroke. Over 11 years of performing this procedure we have observed only one such incident $(0.17 \%)$, in which symptoms largely resolved during follow-up, and one short-lasting transient ischemic attack; this was much less than the stroke rate reported for the RF-based AF ablation procedures (approx. 1\%). However, according to the literature silent cerebral embolization, a phenomenon of unknown clinical significance, occurs in approximately $25 \%$ of patients - similarly as in many other car- diac interventions that involve left heart catheterization. Most likely the cerebral embolization is mainly related to air embolism. Great care is mandatory during sheath management; catheter insertion and withdrawal should be slow to avoid air suction via the Flexcath valve, and reinsertion of the CB catheter should be avoided.

Pericarditis develops in a very small percentage of patients after cryoablation ablation - probably due to a larger than usual surface of the transmural lesions. The longterm consequence of subclinical pericarditis (Figure 11), probably an underestimated phenomenon, could be the formation of pericardial adhesions [35]. Hemodynamically important iatrogenic atrial septal defect (ASD) and its consequences after PVI seem to be an underestimated problem as well. There are limited data from the literature regarding this complication [36]; the large diameter of the Flexcath sheath that facilitates this complication is one of the weak sides of the current technology. Apart from many cases of asymptomatic, tiny ASD and a few cases of ASD requiring percutaneous intervention, we have observed a few cases of severe migraine after $C B$ ablation, a complication probably related to iatrogenic ASD. In some patients this was de novo migraine, but in most cases it was an exacerbation of the pre-existing migraine that was inactive before the procedure, sometimes for many years. In all our cases migraine subsided within a few months. The estimated incidence of migraine after ablation/transseptal puncture is 1-2\% [37].

Palsy of the right phrenic nerve deserves special attention as it remains the Achilles' heel of the cryoballoon technique (Figure 12). It usually occurs during freezing in the right superior PV, less frequently in the right inferior PV. It is the most common intraprocedural complication, occurring in about $8-6 \%$ of patients; although it usually resolves within a few minutes it limits further freeze delivery to the right PVs. Persistent palsy (i.e. still present 

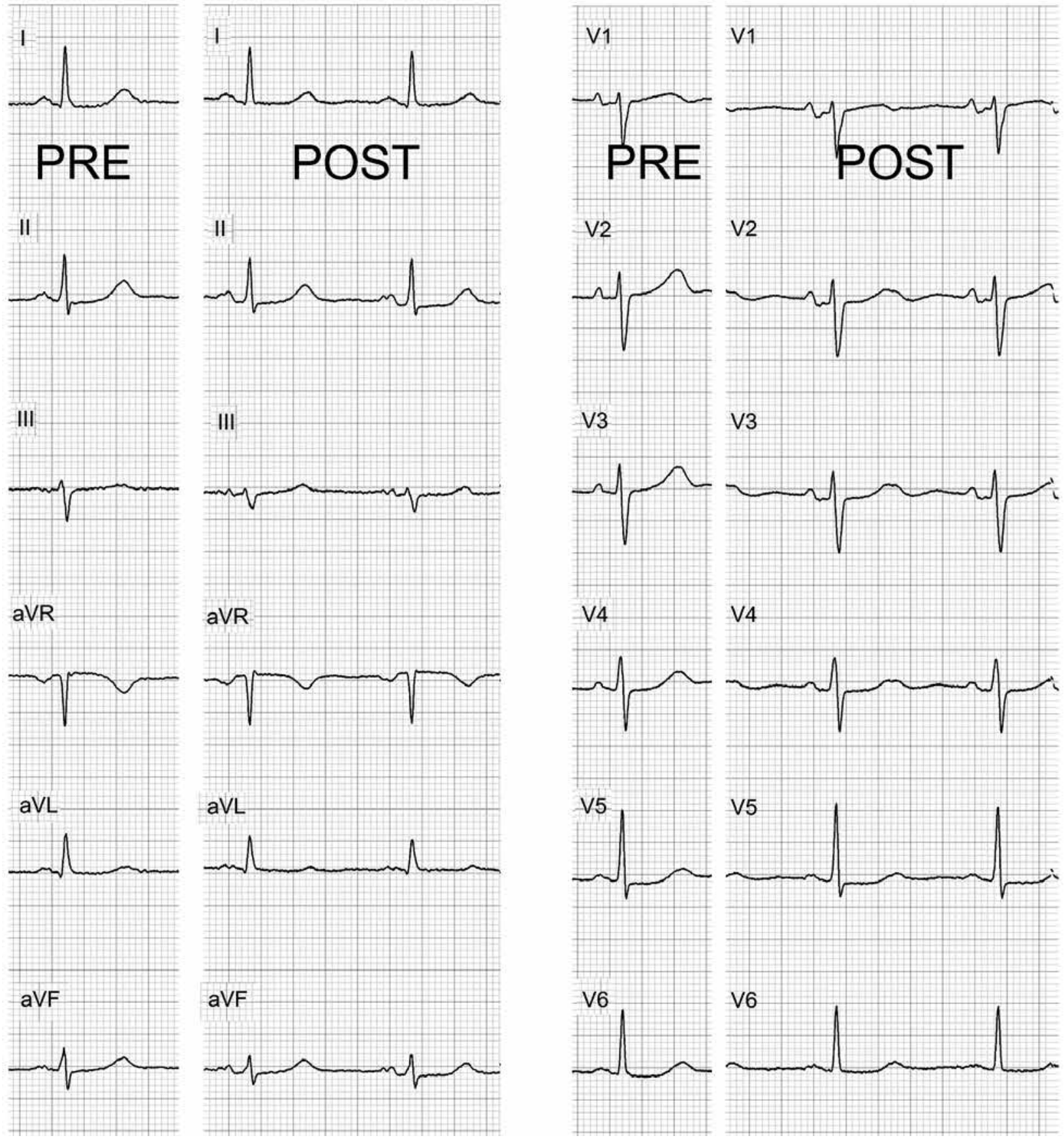

Figure 11. 12-lead ECG recorded at the beginning (panels 'PRE') and the end (panels 'POST') of an uneventful cryoballoon ablation procedure. After the procedure there is an evident depression of the PR interval in several leads (II, III, aVF, V1-V3) as well PR interval elevation in lead aVR indicating pericardial inflammation due to the transmurality of the cryoablation lesions - or more extensive necrosis/damage to the left atrium. This is a common ECG phenomenon post cryoballoon ablation

at discharge) is present in approximately $1-2 \%$ of cases [38]. Steroids might be administered in such cases to limit inflammation. In most of these cases the phrenic nerve regains function within 1 year. Permanent nerve palsy affects approximately $0.2 \%$ of cases, fortunately, most of them remain asymptomatic. A chest X-ray is recommended before the discharge in cases where phrenic nerve palsy was observed during the procedure. In some patients phrenic nerve palsy might course under the mask of pneumonia/atelectasis.

Several precautions can limit this complication significantly. During every freeze in the right sided PVs the phrenic nerve activity must be monitored by constant pacing. The left phrenic nerve can be paced by placing the coronary sinus deflectable catheter near the junction of the superior vena cava and the right subclavian 

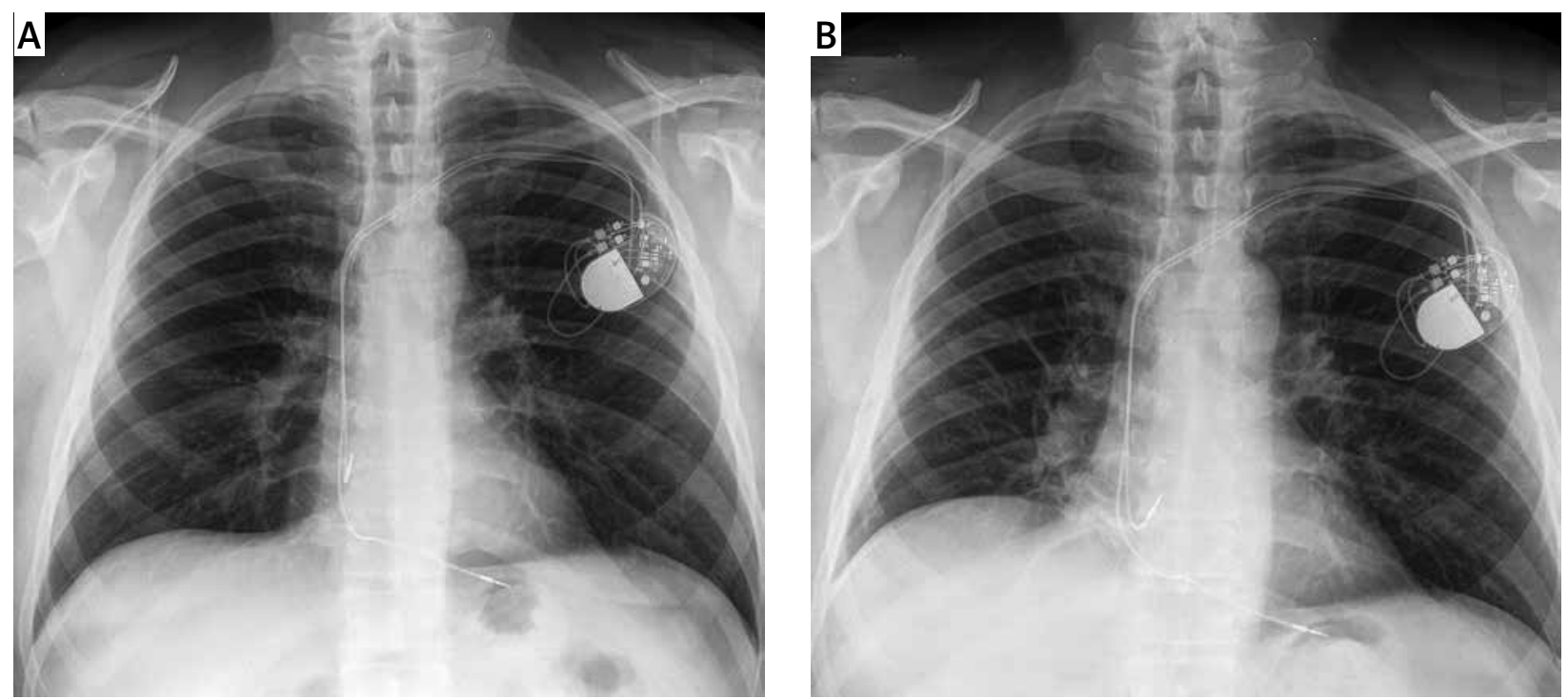

Figure 12. Chest X-ray performed before (upper panel) and after ablation illustrates the most common complication of cryoballoon based pulmonary vein isolation - phrenic nerve palsy. Note the higher position of the right diaphragmatic dome after the procedure

vein. Phrenic nerve function is then monitored by constant palpation of the abdomen. At the first moment when the strength of the diaphragmatic excursion decreases, the balloon should be deflated using the double stop technique (resulting in immediate deflation rather than delayed deflation occurring when the balloon is rewarmed). Some recommend monitoring the diaphragmatic compound motor action potential [39]. This can be easily done by observing ECG with modified lead I: the right-arm electrode is placed above the xiphoid, and the left-arm ECG electrode is placed along the right costal margin. This methods also allows the other members of the team to monitor the nerve function in the electrophysiological laboratory and, according to a few studies, makes it possible to detect the damage to the phrenic nerve at a slightly earlier phase than monitoring mechanical function. We ceased to use this method as in our experience it does not offer any advantage over manual palpation; a noticeable decrease in the potential amplitude seems to be coincident with the decrease in diaphragmatic excursion. Apart from monitoring the nerve function it is important to position the balloon in the PV as proximally as possible, not allowing too low temperatures, and occasionally use the 'pull-back' maneuver.

\section{Conclusions}

Cryoballoon technology greatly simplified the contemporary approach to the PV isolation procedure, without compromising outcomes, offering a new hope for patients with AF. For a safe and effective procedure the electrophysiologist/interventionalist should be aware of all minute details of the procedure including several tricks developed by the most experienced operators and the pre-procedural and post-procedural management recommendations. Detailed knowledge of complications of CB-based ablation is mandatory.

\section{Conflict of interest}

Dr Jastrzębski - moderate lecture and proctoring fees from Medtronic. Other author declare no conflict of interest.

\section{References}

1. Menezes MN, Cortez-Dias N, Carpinteiro L, et al. One-shot ablation for PV isolation. J Atr Fibrillation 2014; 7: 1111.

2. Kuck KH, Fürnkranz A, Chun KJ, et al. Cryoballoon or radiofrequency ablation for symptomatic paroxysmal atrial fibrillation: reintervention, rehospitalization, and quality-of-life outcomes in the FIRE AND ICE trial. Eur Heart J 2016; 37: 2858-65.

3. Friberg L, Tabrizi F, Englund A. Catheter ablation for atrial fibrillation is associated with lower incidence of stroke and death: data from Swedish health registries. Eur Heart J 2016; 37: 2478-87.

4. Srivatsa UN, Danielsen B, Amsterdam EA, et al. CAABL-AF (California Study of Ablation for Atrial Fibrillation) Mortality and Stroke, 2005 to 2013. Circ Arrhythm Electrophysiol 2018; 11: e005739.

5. Jarman JW, Hussain W, Wong T, et al. Resource use and clinical outcomes in patients with atrial fibrillation with ablation versus antiarrhythmic drug treatment. BMC Cardiovasc Disord 2018; 18: 211.

6. Peigh G, Kaplan RM, Bavishi A, et al. A novel risk model for very late return of atrial fibrillation beyond 1 year after cryoballoon ablation: the SCALE-CryoAF score. J Interv Card Electrophysiol 2020; 58: 209-17.

7. Calkins H, Hindricks G, Cappato R, et al. 2017 HRS/EHRA/ECAS/ APHRS/SOLAECE expert consensus statement on catheter and surgical ablation of atrial fibrillation. Europace 2018; 20: e1-60.

8. Kawaguchi N, Okishige K, Yamauchi Y, et al. Predictors of a persistent status of pulmonary vein electrical isolation by a cryo- 
balloon application for drug-refractory atrial fibrillation. Circ J 2018; 82: 659-65.

9. Ahmed H, Neuzil P, Skoda JA, et al. The permanency of pulmonary vein isolation using a balloon cryoablation catheter. J Cardiovasc Electropsyhiol 2010; 21: 731-7.

10. Wei HQ, Li J, Sun Q, et al. Safety and efficacy of superior vena cava isolation using the second-generation cryoballoon ablation in a canine model. J Cardiol 2020; 75: 368-73.

11. Yorgun $\mathrm{H}$, Canpolat $\mathrm{U}$, Okşul M, et al. Long-term outcomes of cryoballoon-based left atrial appendage isolation in addition to pulmonary vein isolation in persistent atrial fibrillation. Europace 2019; 21: 1653-62.

12. Niazi I, Erickson L, Chaudhari A, Djelmami-Hani M. Cryoballoon pulmonary vein isolation and roof and posterior wall debulking using Navik 3D ${ }^{\mathrm{TM}}$ : a new technique for atrial fibrillation ablation. J Innov Card Rhythm Manag 2020; 11: 3975-82.

13. Kusiak A, Jastrzębski M, Bednarski A, et al. Diagnostic value of implantable loop recorder in patients undergoing cryoballoon ablation of atrial fibrillation. Ann Noninvasive Electrocardiol 2020; 25: e12733.

14. Jain SK, Novak PG, Sangrigoli R, et al. Sustained quality-of-life improvement post-cryoballoon ablation in patients with paroxysmal atrial fibrillation: results from the STOP-AF Post-Approval Study. Heart Rhythm 2020; 17: 485-91.

15. Aryana A, Singh SM, Kowalski M, et al. Acute and long-term outcomes of catheter ablation of atrial fibrillation using the second-generation cryoballoon versus open-irrigated radiofrequency: a multicenter experience. J Cardiovasc Electropsyhiol 2015; 26: 832-9.

16. Mörtsell D, Arbelo E, Dagres N, et al. Cryoballoon vs. radiofrequency ablation for atrial fibrillation: a study of outcome and safety based on the ESC-EHRA atrial fibrillation ablation longterm registry and the Swedish catheter ablation registry. Europace 2019; 21: 581-9.

17. Canpolat $\mathrm{U}$, Kocyigit $\mathrm{D}$, Yalcin $\mathrm{MU}$, et al. Long-term outcomes of pulmonary vein isolation using second-generation cryoballoon during atrial fibrillation ablation. PACE 2019; 42: 910-21.

18. Ravi V, Poudyal A, Pulipati P, et al. A systematic review and meta-analysis comparing second-generation cryoballoon and contact force radiofrequency ablation for initial ablation of paroxysmal and persistent atrial fibrillation. J Cardiovasc Electropsyhiol 2020; 31: 2559-71.

19. Pruszkowska P, Lenarczyk R, Gumprecht J, et al. Cryoballoon ablation of atrial fibrillation in patients with advanced systolic heart failure and cardiac implantable electronic devices. Kardiol Pol 2018; 76: 1081-8.

20. Heeger $\mathrm{CH}$, Abdin A, Mathew S, et al. Efficacy and safety of cryoballoon ablation in patients with heart failure and reduced left ventricular ejection fraction - a multicenter study. Circ J 2019; 83: 1653-9.

21. Maj R, lacopino S, Ströker E, et al. Mid-term outcome following second-generation cryoballoon ablation for atrial fibrillation in heart failure patients: effectiveness of single 3-min freeze cryoablation performed in a cohort of patients with reduced left ventricular systolic function. J Cardiovasc Med 2019; 20: 667-75.

22. Marrouche NF, Brachmann J, Andresen D, et al. Catheter ablation for atrial fibrillation with heart failure. N Engl J Med 2018; 378: 417-27.

23. Jastrzębski $M$, Kiełbasa G, Fijorek K, et al. Outcomes of single-shot techniques based atrial fibrillation ablation program. Adv Interv Cardiol 2020; 16: 466-73.
24. Straube F, Dorwarth U, Ammar-Busch S, et al. First-line catheter ablation of paroxysmal atrial fibrillation: outcome of radiofrequency vs. cryoballoon pulmonary vein isolation. Europace 2016; 18: 368-75.

25. Liu XH, Gao XF, Jin CL, et al. Cryoballoon versus radiofrequency ablation for persistent atrial fibrillation: a systematic review and meta-analysis. Kardiol Pol 2019; 78: 20-9.

26. Andrade JG, Champagne J, Dubuc M, et al. Cryoballoon or radiofrequency ablation for atrial fibrillation assessed by continuous monitoring: a randomized clinical trial. Circ 2019; 140: 1779-88.

27. Hoffmann E, Straube F, Wegscheider K, et al. Outcomes of cryoballoon or radiofrequency ablation in symptomatic paroxysmal or persistent atrial fibrillation. Europace 2019; 21: 1313-24.

28. Rottner L, Fink T, Heeger CH, et al. Is less more? Impact of different ablation protocols on periprocedural complications in second-generation cryoballoon based pulmonary vein isolation. Europace 2018; 20: 1459-67.

29. Kuck KH, Brugada J, Fürnkranz A, et al. Cryoballoon or radiofrequency ablation for paroxysmal atrial fibrillation. $N$ Engl J Med 2016; 374: 2235-45.

30. Luik A, Radzewitz A, Kieser M, et al. Cryoballoon versus open irrigated radiofrequency ablation in patients with paroxysmal atrial fibrillation: the prospective, randomized, controlled, noninferiority FreezeAF study. Circ 2015; 132: 1311-9.

31. Mark DB, Anstrom KJ, Sheng S, et al. Effect of catheter ablation vs medical therapy on quality of life among patients with atrial fibrillation: the CABANA randomized clinical trial. JAMA 2019; 321: 1275-85.

32. Bollmann A, Ueberham L, Schuler E, et al. Cardiac tamponade in catheter ablation of atrial fibrillation: German-wide analysis of 21141 procedures in the Helios atrial fibrillation ablation registry (SAFER). Europace 2018; 20: 1944-51.

33. Canpolat U, Kocyigit D, Aytemir K. Complications of atrial fibrillation cryoablation. J Atr Fibrillation 2017; 10: 1620.

34. John RM, Kapur S, Ellenbogen KA, Koneru JN. Atrioesophageal fistula formation with cryoballoon ablation is most commonly related to the left inferior pulmonary vein. Heart Rhythm 2017; 14: 184-9.

35. Velagic V, de Asmundis C, Chierchia GB, La Meir M. Pericardial adhesions as a consequence of cryoballoon ablation detected during the hybrid AF ablation procedure. Heart Rhythm Case Reports 2016; 2: 111-2.

36. Watanabe T, Miyazaki S, Kajiyama T, et al. Persistence of an iatrogenic atrial septal defect after a second-generation cryoballoon ablation of atrial fibrillation. Heart Vessels 2018; 33: 1060-7.

37. Kato Y, Hayashi T, Kato R, Takao M. Migraine-like headache after transseptal puncture for catheter ablation: a case report and review of the literature. Intern Med 2019; 58: 2393-95.

38. Miyazaki S, Kajiyama T, Watanabe T, et al. Characteristics of phrenic nerve injury during pulmonary vein isolation using a 28$\mathrm{mm}$ second-generation cryoballoon and short freeze strategy. J Am Heart Assoc 2018; 7: e008249.

39. Su W, Kowal R, Kowalski M, et al. Best practice guidelines for cryoballoon ablation in atrial fibrillation: the compliation experience of more than 3000 procedures. Heart Rhythm 2015; 12: 1658-66. 